\title{
Optical lattice-like cladding waveguides by direct laser writing: fabrication, luminescence, and lasing
}

\author{
Weijie Nie, ${ }^{1}$ Ruiyun He, ${ }^{1}$ Chen Cheng, ${ }^{1}$ Uéslen Rocha, ${ }^{2}$ Javier Rodríguez Vázquez de Aldana, ${ }^{3}$ \\ Daniel Jaque, ${ }^{2}$ and Feng Chen ${ }^{1, *}$ \\ ${ }^{1}$ School of Physics, State Key Laboratory of Crystal Materials, Shandong University, Jinan 250100, China \\ ${ }^{2}$ Fluorescence Imaging Group, Departamento de Física de Materiales, Facultad de Ciencias, Universidad Autónoma de Madrid, \\ Madrid 28049, Spain \\ ${ }^{3}$ Laser Microprocessing Group, Facultad Ciencias, Universidad de Salamanca, Salamanca 37008, Spain \\ ${ }^{*}$ Corresponding author: drfchen@sdu.edu.cn
}

Received 4 February 2016; revised 13 March 2016; accepted 6 April 2016; posted 7 April 2016 (Doc. ID 258832); published 3 May 2016

We report on the fabrication of optical lattice-like waveguide structures in an Nd:YAP laser crystal by using direct femtosecond laser writing. With periodically arrayed laserinduced tracks, the waveguiding cores can be located in either the regions between the neighbored tracks or the central zone surrounded by a number of tracks as outer cladding. The polarization of the femtosecond laser pulses for the inscription has been found to play a critical role in the anisotropic guiding behaviors of the structures. The confocal photoluminescence investigations reveal different stress-induced modifications of the structures inscribed by different polarization of the femtosecond laser beam, which are considered to be responsible for the refractive index changes of the structures. Under optical pump at $808 \mathrm{~nm}$, efficient waveguide lasing at $\sim 1 \mu \mathrm{m}$ wavelength has been realized from the optical lattice-like structure, which exhibits potential applications as novel miniature light sources. (c) 2016 Optical Society of America

OCIS codes: (230.7370) Waveguides; (140.3390) Laser materials processing; (130.3120) Integrated optics devices.

http://dx.doi.org/10.1364/OL.41.002169

Waveguide-based optical lattices receive considerable attention for their applications in nonlinear optics and quantum photonics [1-5]. Such lattice structures, usually in the form of waveguide arrays, can be fabricated by a few techniques in optical materials. For example, by applying writing of optical spatial solitons, 2D optical lattice with quadrangular geometry was produced in a photorefractive crystal [6-8]. Recently, femtosecond laser writing has become a powerful technique to produce $3 \mathrm{D}$ photonic devices due to its unique capability of $3 \mathrm{D}$ engineering in micrometric or even nanometer scales, the excellent advantages of negligible thermal-diffusion effect, and wide applicability of optical materials [9-14]. As first demonstrated by Davis et al. in 1996 in glasses [15], femtosecond laser writing has been utilized for manufacturing guiding devices in many transparent materials, such as dielectric crystals, ceramic, and organic materials, to realize applications in diverse disciplines [16-20]. The focused femtosecond laser pulses induce extremely localized modification of permanent or stable refractive-index changes through nonlinear processes [21]. In glasses, femtosecond laser pulses usually induce positive changes $(\Delta n>0)$ of the refractive index to form the waveguide located inside the focal volume, while in crystalline media negative index changes $(\Delta n<0)$ are typically induced at the focal region, but a positive index change can be produced in the surroundings; such modifications can be used to fabricate efficient waveguides by the dual-line strategy (waveguide between two parallel laser damage lines) or by depressed cladding inscription (undamaged core surrounded by a large number of damage tracks) $[20,22]$. In both structures with negative index change at the damage tracks, the performances of original properties of the crystal are well preserved in the waveguide because the waveguide core locates in a volume that is not modified by the laser pulses. For instance, during the fabrication of waveguides in rare-earth ion-doped crystals, the waveguide keeps the spectroscopic properties of the active ions, showing excellent performances as waveguide lasers [17,23-25]. An alternative waveguide fabrication technique in crystalline materials that is based on hexagonal lattices of laser induced damage tracks has been recently demonstrated. Such waveguides, implemented on an Nd:YAG laser crystal, allowed efficient light-guiding and beam tailoring, and is an excellent candidate for constructing complex integrated laser circuits [26].

In an Nd:YAG crystal (isotropic), the inscribed optical lattices, which behave as cladding waveguides, are only used for confining the beam in the core [26]. However, in anisotropic crystals, the function of guiding or confining of the optical lattice can be realized with a variation of the inscription-laser polarization. In this work, a typical birefringent laser crystal $\mathrm{Nd}$ :YAP (neodymium-doped yttrium aluminum perovskite) is chosen for investigating optical-lattice structures owing to its 
high thermal conductivity, excellent optomechanical coefficient, and great stimulated emission cross section [27,28]. Benefiting from the large natural birefringence of Nd:YAP, the gain coefficient and polarization of stimulated emission cross section can be varied with the crystallographic orientation of the laser crystal, which offers the possibility of optimizing particular performance characteristics [29]. Previously, tunable dualwavelength lasers from depressed cladding waveguides have been studied in b-cut Nd:YAP crystals by changing the pump polarization corresponding to the variation of crystallographic orientation of the laser crystal [30]. Similarly, when the polarization of the irradiation femtosecond laser is changed to corresponding orthometric crystallographic orientation of the laser crystal, it is possible to obtain not only a confining lattice but also a guiding lattice. In addition, due to the synergy effect of the physical nature of the materials and laser pulse parameters for refractive index changes, many parameters that determine the morphology, size, and type of the modification by the laser pulse have been reported, such as pulse energy, pulse duration, repetition rate of the laser pulse, scanning velocity, and focusing condition [20]. However, the influence of polarization, which is another crucial parameter for femtosecond laser fabricated waveguides, has been scarcely reported [31]. In this Letter, we report on the fabrication of the optical lattice-like waveguide structures in Nd:YAP laser crystals by using different polarizations of femtosecond lasers. The fluorescence and guiding properties have been measured to reveal the waveguide nature induced by femtosecond lasers. The continuous waveguide laser at $\sim 1 \mu \mathrm{m}$ is generated with an optical pump at $812 \mathrm{~nm}$.

Figure 1 depicts the femtosecond laser inscription setup that was utilized for the fabrication in an optically polished $\mathrm{Nd}$ :YAP crystal with the dimension of 10 (c) $\mathrm{mm} \times 10$ (b) $\mathrm{mm} \times$ 2(a) $\mathrm{mm}$. The laser source was an amplified Ti:sapphire femtosecond system (Spitfire, Spectra Physics) that delivers linearly polarized pulses with a temporal duration of $120 \mathrm{fs}$, a central wavelength of $795 \mathrm{~nm}$, and a repetition-rate of $1 \mathrm{kHz}$. The pulse energy (up to $1 \mathrm{~mJ}$ ) was tuned by a calibrated neutral density filter (NDF) placed after a set of half-wave plate and linear-polarizing cube, which can be used to change the polarization of the femtosecond laser. The beam is then focused through one of the $10 \mathrm{~mm} \times 10 \mathrm{~mm}$ sample surfaces by a $20 \times$ microscope objective $(\mathrm{NA}=0.40)$ with a pulse energy of $0.84 \mu \mathrm{J}$. The sample, located at a $3 \mathrm{D}$-motorized stage, is

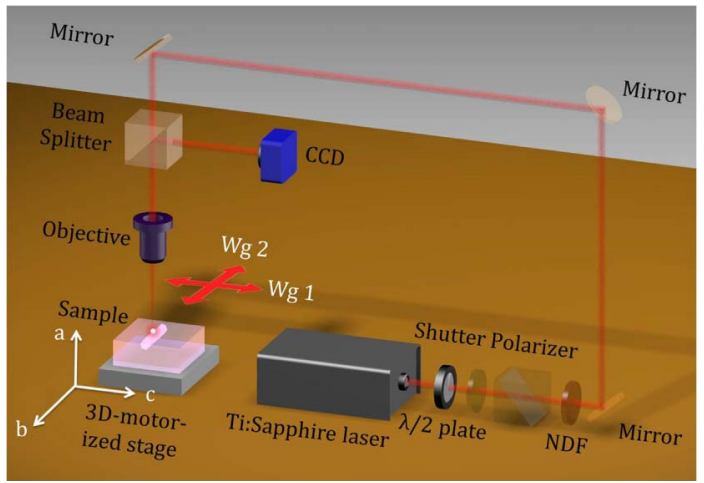

Fig. 1. Schematic plot of optical lattice-like waveguide structure fabrication by femtosecond laser inscription. The orthogonal red arrows represent the polarization direction of the femtosecond laser in the waveguides.

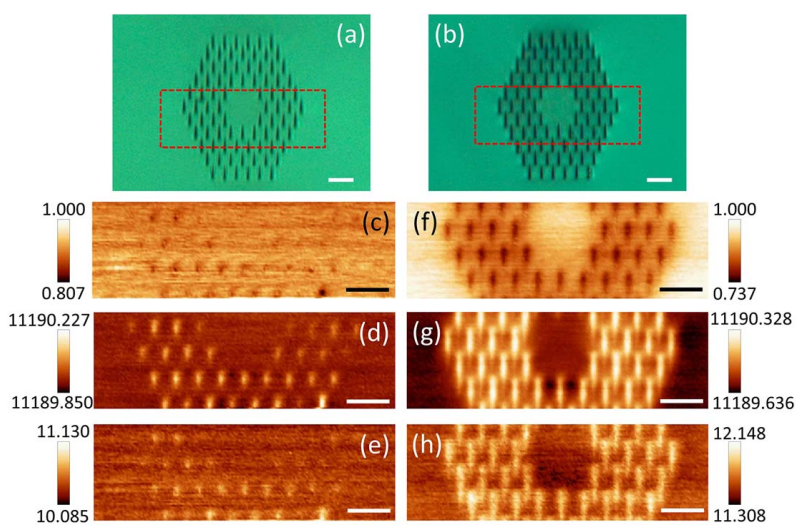

Fig. 2. Microscope cross-sectional images of the photonic latticelike cladding waveguides (a) No. 1 and (b) No. 2. The red dotted rectangles display the regions for the confocal micro-photoluminescence measurements. The spatial distributions of (c) emitted intensity, (d) spectral shift, and (e) linewidth obtained from the end face of the photonic lattice-like cladding waveguide No. 1. (f), (g), and (h) correspond to waveguide No. 2, respectively. The scale bars in the bottom right of all images are $20 \mu \mathrm{m}$.

scanned at a constant velocity of $0.5 \mathrm{~mm} / \mathrm{s}$ along a $b$-axis orientation, thus forming damage tracks with a transverse length of $10 \mu \mathrm{m}$. Several parallel scans are performed at different positions of the sample, following the desired hexagonal geometry with a lateral separation of $10 \mu \mathrm{m}$ between each of two adjacent damage tracks. Under this condition, two optical lattice-like waveguide structures are fabricated with exactly the same parameters, except for the polarization direction of the femtosecond laser, which is shown in Fig. 1 with the orthogonal red arrows. Figures 2(a) and 2(b) depict the optical microscope cross-sectional images of waveguide No. 1, with the polarization perpendicular to the scans, and waveguide No. 2, with the polarization parallel to the scans, respectively.

The characterization of the waveguides is done in terms of fluorescence and guidance. For the fluorescence analysis, a 488-nm CW diode laser is used as excitation source, which is focused by a $50 \times$ microscope objective $(\mathrm{NA}=0.55)$. The subsequent emission generated by $\mathrm{Nd}^{3+}$ ions is collected by the same microscope objective and is spectrally analyzed by a highresolution spectrometer. The crystal is mounted on a $2 \mathrm{D}$ motorized stage with a spatial resolution of $100 \mathrm{~nm}$ to acquire the per-point emission spectrum, which is analyzed and fitted by the software LabSpec. Finally, the different fluorescence properties of spectral position, width, and intensity of fluorescence lines are processed by using the software WSMP [32]. For the guiding performance of the different fabricated structures, a typical end-coupling system is employed with a linearly polarized CW solid-state laser at a wavelength of 1064-nm. The near-field modal distributions are imaged by an infrared CCD camera, and the polarization guiding properties are characterized by a power meter with a change in the polarization of input light. The propagation losses of the structures are estimated and determined by directly measuring the input and output power of the transmitted light.

The waveguide laser is excited in optical lattice-like waveguide structure No. 1. The pump source generated from a tunable CW Ti:sapphire laser (Coherent MBR PE) is a linearly polarized beam with a waist radius of approximately $0.75 \mathrm{~mm}$ 
at $812 \mathrm{~nm}$. A spherical convex lens with a focal length of $25 \mathrm{~mm}$ is used to couple the pump light into the waveguide with an efficiency of 0.81 . A pair of dielectric-coupled mirrors is employed to construct the Fabry-Perot lasing resonant cavity by a set of mechanical fixtures. Under this condition, an input mirror with high transmission ( 98\%) at $0.81 \mu \mathrm{m}$ and high reflectivity $(\sim 99 \%)$ at $1.07 \mu \mathrm{m}$ is adhered to the polished input facet, while an output one with reflectivity of $99 \%$ at $0.81 \mu \mathrm{m}$ and $80 \%$ at $1.07 \mu \mathrm{m}$ is closely attached with the polished output facet. The generated laser is collected by a $20 \times$ microscope objective lens $(\mathrm{NA}=0.4)$ from the output facet of structure.

Figures 2(c)-2(e) and 2(f)-2(h) demonstrate the fluorescence images (corresponding to spatial distribution of emitted intensity, spectral shift, and linewidth) of the ${ }^{4} \mathrm{~F}_{3 / 2} \rightarrow{ }^{4} \mathrm{I}_{9 / 2}$ emission line at $\sim 893 \mathrm{~nm}$, as obtained from the scanning area (indicated by the dashed lines with rectangular geometry) crossing the core of structures which contain the detailed properties of both tracks and core regions, schematically depicted in Figs. 2(a) and 2(b). The fluorescence images are measured in order to get a complete knowledge of the micromodification changes induced by ultrafast irradiation. It can be found that the microstructural lattices change slightly in both structures. Intensity maps reveal that damage creation (denoted by local reductions in the luminescence intensity) is more relevant in waveguide No. 2 than in No. 1. This fact, together with the lower disorder revealed by the low contrast in the width-based image, suggest that fabrication parameters lead to a larger damage in waveguide No. 2 than in No. 1. On one hand, the luminescence images based on spectral shifts reveal a more localized and defined elastically modified areas in waveguide No. 1 than in No. 2. All these maps suggest that fabrication parameters used in waveguide No. 1 created a better defined strain pattern with a reduced damage level. In these conditions, it can be concluded that waveguide No. 2 is constituted by an unaffected core surrounded by a pseudocontinuous damage (low refractive index) core. On the other hand, waveguide No. 1 is constituted by a well-defined compressed/dilated 2D lattice so that waveguiding is expected in this area rather than at the central (core) volume. Note at this point that intensity reduction is here unequivocally related to the existence of local damage as other effects (such as $\mathrm{Nd}^{3+}$ ion migration) can be ignored for the repetition rates used in this work that lead to negligible heat accumulation. This is, indeed, what was observed in the beam propagation experiments, as is explained next.

Figures 3(a) and 3(b) demonstrate the measured near-field modal profiles at $1064 \mathrm{~nm}$ in optical lattice-like waveguide structure No. 1 with TM and TE polarization, respectively. As can be seen, the waveguide No. 1 with the fs-laser polarization perpendicular to the scans is located in the region between two tracks instead of that inside the tracks. For structure No. 2, at the wavelength of $1064 \mathrm{~nm}$, the mode distributions at TM and TE direction are shown in Figs. 3(c) and $3(\mathrm{~d})$, respectively. Different from structure No. 1, the waveguiding region is in the unmodified core surrounded by a hexagonally arrayed track lattice. It should be mentioned that guiding properties are all in good agreement with fluorescence analysis. In addition, both waveguides support well-confined modes along both TM and TE polarization. In order to achieve thorough information on the polarization effect on transmission, the all-angle light guidance along the transverse plane is measured at $1064 \mathrm{~nm}$, and results are shown in Fig. 4.

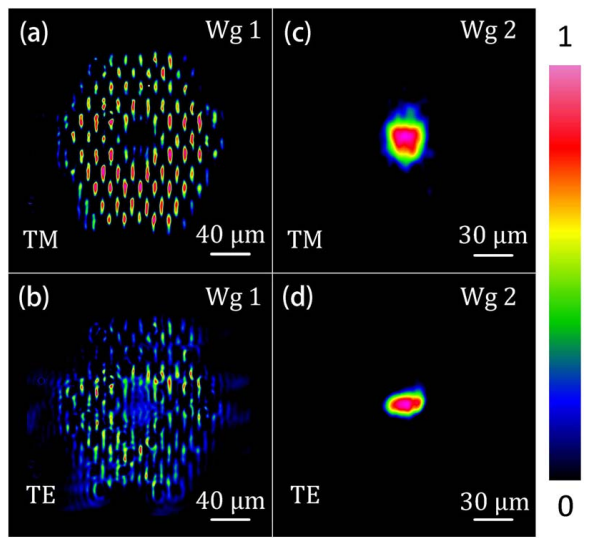

Fig. 3. Measured near-field modal profiles along both (a) TM and (b) TE polarizations at $1064 \mathrm{~nm}$ in structures No. 1. (c) and (d) correspond to No. 2, respectively.

With the identical input power of $135 \mathrm{~mW}$, the performances of both structures are clearly different. For waveguide No. 1, the transmitted power is almost the same for any polarization of input light, which is better than the reported simple dual-line waveguides fabricated in other crystals (e.g., Nd:YAG and Nd: GGG) only supporting the TM-guided modes [18]. For waveguide No. 2, the transmitted power changes by rotating the polarization of the input laser. The maximum transmitted power appears at $\theta=90^{\circ}$ (i.e., TM polarization), while the minimum one occurs at $\theta=0^{\circ}$ (i.e., TE polarization), which indicates the anisotropy effect induced by the femtosecond laser irradiation. Eventually, as one of the essential factors for waveguide transmission, the propagation attenuations of optical lattice-like waveguide structures No. 1 and No. 2 are measured, respectively. It is determined to be $\sim 0.60 \mathrm{~dB}$ and $\sim 0.52 \mathrm{~dB}$ in structure No. 1 at TM and TE polarization, which shows excellent guiding properties at two orthogonal polarizations, while the obtained losses for structure No. 2 are $\sim 3.23 \mathrm{~dB}$ and $\sim 7.84 \mathrm{~dB}$ along TM and TE polarization, respectively.

The excellent performance of waveguide No. 1 suggests its potential application for fabrication of compact photonic devices. In this Letter, the direct-pump waveguide laser, which can be applied to on-demand miniature light sources, has been realized. Figure 5 displays the measured laser power as a function of launched power at $808 \mathrm{~nm}$ pump laser in optical lattice-like

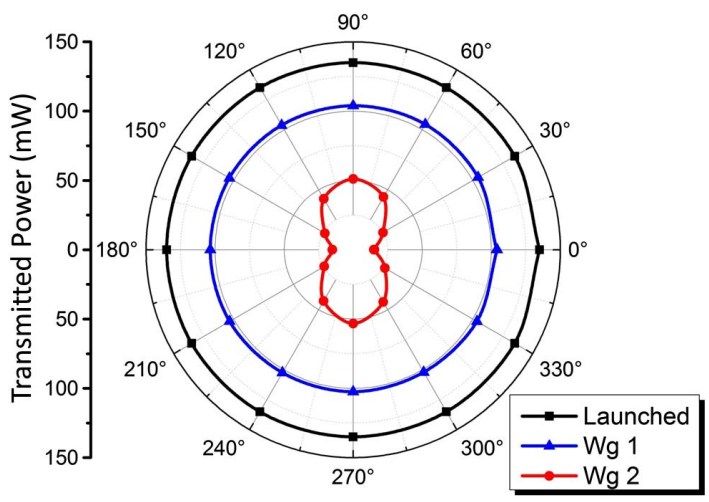

Fig. 4. Transmitted power of waveguide No. 1 (blue circle) and No. 2 (red curve) as a function of all-angle light transmission with the same launched pump power (black circle) at $1064 \mathrm{~nm}$. 


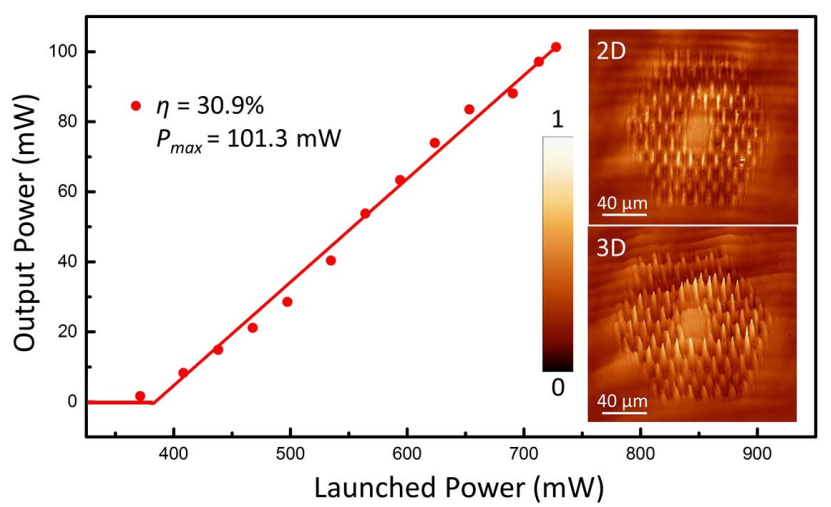

Fig. 5. Output power as a function of launched pump power in optical lattice-like waveguide structure No. 1. The inserts are 2D and $3 \mathrm{D}$ near-field profiles of the generated lasers.
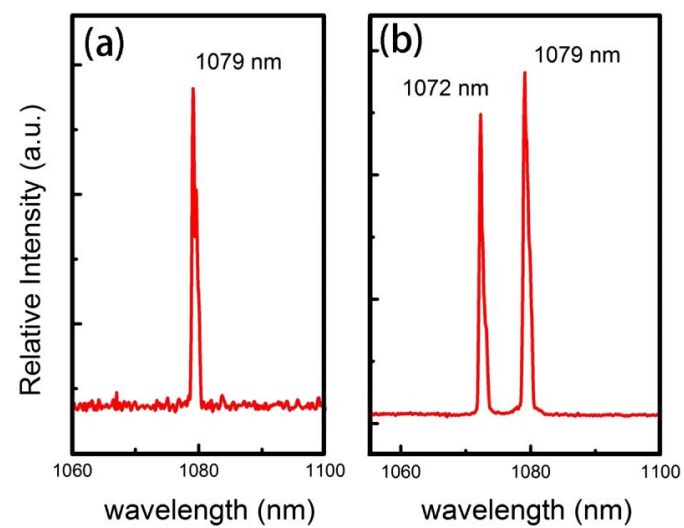

Fig. 6. Spectra of laser emissions (a) at single wavelength of $1079 \mathrm{~nm}$ and (b) at the simultaneous dual-wavelength of 1072 and $1079 \mathrm{~nm}$ in optical lattice-like waveguide structure No. 1.

waveguide structure No. 1. The lasing oscillation begins with the launched power, which exceeds the threshold of $384.5 \mathrm{~mW}$. As the pump power increases linearly to the maximum of $727.7 \mathrm{~mW}$, the output power reaches the maximum value of $101.3 \mathrm{~mW}$, with a slope efficiency of $30.9 \%$. The excited laser spectra are shown in Fig. 6, which correspond to the main emission line of $\mathrm{Nd}^{3+}$ ion ${ }^{4} \mathrm{~F}_{3 / 2} \rightarrow{ }^{4} \mathrm{I}_{11 / 2}$ transition. With a slight change of the incidence point, the spectrum is either at single wavelength of $1079 \mathrm{~nm}$ [i.e., Fig. 6(a)] or at the simultaneous dual-wavelength of 1072 and $1079 \mathrm{~nm}$ [i.e., Fig. 6(b)], a result that was previously reported in [30]. In addition, the similar laser performance but with higher thresholds has been found in bulk. This demonstrates that the laser properties have been well preserved in the waveguides with the advantages of lower lasing thresholds.

In conclusion, optical lattice-like waveguide structures have been successfully fabricated by direct femtosecond laser writing. The effect of the polarization of irradiation femtosecond laser on the fabricated structures has been studied in terms of microstructure analysis and guiding behavior. Microstructure changes are shown in the fluorescence images, revealing that different waveguide types are formed with different polarizations of the irradiation femtosecond laser. In addition, the application of structure No. 1 has been realized for direct-pump waveguide laser at dual-wavelengths of 1072 and $1079 \mathrm{~nm}$. The maximum output power through optical lattice-like waveguide structure No. 1 is $101.3 \mathrm{~mW}$ with a slope efficiency of $30.9 \%$, under the optical pump at $812 \mathrm{~nm}$.

Funding. National Natural Science Foundation of China (NSFC) (11274203); Ministerio de Economia y Competitividad (MINECO) (FIS2013-44174-P, MAT201021270-C04-01).

\section{REFERENCES}

1. J. W. Fleischer, M. Segev, N. K. Efremidis, and D. N. Christodoulides, Nature 422, 147 (2003).

2. H. B. Perets, Y. Lahini, F. Pozzi, M. Sorel, R. Morandotti, and Y. Silberberg, Phys. Rev. Lett. 100, 170506 (2008).

3. D. N. Christodoulides, F. Lederer, and Y. Silberberg, Nature 424, 817 (2003).

4. M. Gräfe, A. S. Solntsev, R. Keil, A. A. Sukhorukov, M. Heinrich, A. Tünnermann, S. Nolte, A. Szameit, and Yu. S. Kivshar, Sci. Rep. 2 562 (2012).

5. T. J. Alexander and Y. S. Kivshar, Appl. Phys. B 82, 203 (2006).

6. W. Chen, X. Zhu, T. Wu, and R. Li, Opt. Express 18, 10956 (2010).

7. N. K. Efremidis, J. Hudock, D. N. Christodoulides, J. W. Fleischer, O. Cohen, and M. Segev, Phys. Rev. Lett. 91, 213906 (2003).

8. Y. V. Kartashov, R. Carretero-González, B. A. Malomed, V. A. Vysloukh, and L. Torner, Opt. Express 13, 10703 (2005).

9. K. Sugioka and Y. Cheng, Light Sci. Appl. 3, e149 (2014).

10. M. Ams, G. D. Marshall, P. Dekker, J. A. Piper, and M. J. Withford, Laser Photon. Rev. 3, 535 (2009).

11. D. Choudhury, J. R. Macdonald, and A. K. Kar, Laser Photon. Rev. 8 827 (2014).

12. R. R. Gattass and E. Mazur, Nat. Photonics 2, 219 (2008).

13. S. Müller, T. Calmano, P. Metz, N.-O. Hansen, C. Kränkel, and G. Huber, Opt. Lett. 37, 5223 (2012).

14. J. Siebenmorgen, T. Calmano, K. Petermann, and G. Huber, Opt. Express 18, 16035 (2010).

15. K. M. Davis, K. Miura, N. Sugimoto, and K. Hirao, Opt. Lett. 21, 1729 (1996).

16. K. Sugioka, J. Xu, D. Wu, Y. Hanada, Z. Wang, Y. Cheng, and K. Midorikawa, Lab Chip 14, 3447 (2014).

17. G. Salamu, F. Jipa, M. Zamfirescu, and N. Pavel, Opt. Express 22, 5177 (2014).

18. H. Liu, J. R. Vázquez de Aldana, M. Hong, and F. Chen, IEEE J. Sel. Top. Quantum Electron. 22, 4500204 (2016).

19. Q. Liu, S. Gross, P. Dekker, M. J. Withford, and M. J. Steel, Opt. Express 22, 28037 (2014).

20. F. Chen and J. R. Vázquez de Aldana, LaserPhoton. Rev. 8, 251 (2014).

21. B. C. Stuart, M. D. Feit, S. Herman, A. M. Rubenchik, B. W. Shore, and M. D. Perry, Phys. Rev. B 53, 1749 (1996).

22. F. Chen and J. R. Vázquez de Aldana, Proc. SPIE 9532, 95320M (2015).

23. C. Grivas, Prog. Quantum Electron. 35, 159 (2011).

24. W. F. Silva, C. Jacinto, A. Benayas, J. R. Vázquez de Aldana, G. A. Torchia, F. Chen, Y. Tan, and D. Jaque, Opt. Lett. 35, 916 (2010).

25. C. Grivas, C. Corbari, G. Brambilla, G. Pavlos, and P. G. Lagoudakis, Opt. Lett. 37, 4630 (2012).

26. Y. Jia, C. Cheng, J. R. Vázquez de Aldana, G. R. Castillo, B. del Rosal Rabes, Y. Tan, D. Jaque, and F. Chen, Sci. Rep. 4, 5988 (2014).

27. H. Shen, T. Lian, R. Zeng, Y. Zhou, and G. Yu, IEEE J. Quantum Electron. 25, 144 (1989).

28. Y. Lü, J. Xia, J. Zhang, X. Fu, and H. Liu, Appl. Opt. 53, 5141 (2014)

29. G. A. Massey, Appl. Phys. Lett. 17, 213 (1970).

30. W. Nie, C. Cheng, Y. Jia, C. Romero, J. R. Vázquez de Aldana, and F. Chen, Opt. Lett. 40, 2437 (2015).

31. B. McMillen and Y. Bellouard, Opt. Express 23, 86 (2015).

32. I. Horcas, R. Fernandez, J. M. Gomez-Rodriguez, J. Colchero, J. Gomez-Herrero, and A. M. Baro, Rev. Sci. Instrum. 78, 013705 (2007). 